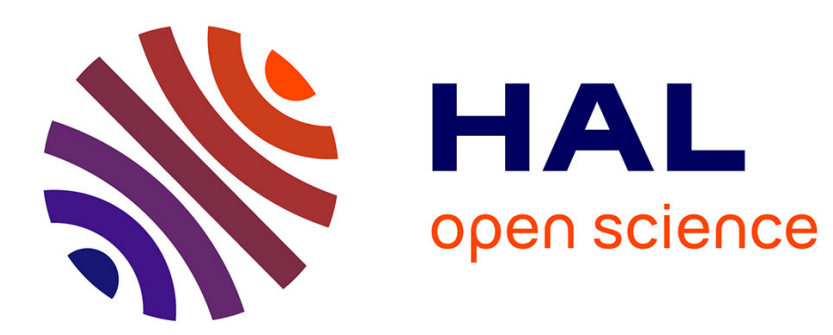

\title{
Elements of Similarity and Singularity
}

Alberto Verga

\section{To cite this version:}

Alberto Verga. Elements of Similarity and Singularity. J.-A. Sepulchre and J. L. Beaumont. Peyresq Lectures on Nonlinear Phenomena, Word Scientific, Singapore, pp.285-318, 2003, Volume 2. hal00092025

\section{HAL Id: hal-00092025 \\ https://hal.science/hal-00092025}

Submitted on 8 Sep 2006

HAL is a multi-disciplinary open access archive for the deposit and dissemination of scientific research documents, whether they are published or not. The documents may come from teaching and research institutions in France or abroad, or from public or private research centers.
L'archive ouverte pluridisciplinaire HAL, est destinée au dépôt et à la diffusion de documents scientifiques de niveau recherche, publiés ou non, émanant des établissements d'enseignement et de recherche français ou étrangers, des laboratoires publics ou privés. 


\title{
ELEMENTS OF SIMILARITY AND SINGULARITY
}

\author{
ALBERTO VERGA \\ Institut de Recherche sur les Phénomènes Hors Équilibre, \\ 49, rue F. Joliot-Curie, BP 146, 13384 Marseille, France \\ E-mail: Alberto.Verga@irphe.univ-mrs.fr
}

\begin{abstract}
We briefly introduce through examples taken from the physics of fluids and continuous media, the concepts of self-similarity and singularity. We start with the elementary formulation of dimensional analysis and its application to several problems in lubrication and nonlinear diffusion. We also treat the more subtle case of second type similarity laws, related to the appearance of anomalous dimensions using a dynamical renormalization group approach. Singularities in physical systems are presented using the example of shock formation for the simple wave equation. The method of characteristics is explained and the similarity solutions are related to the weak solutions of quasilinear equations using the dissipationless limit of the Burgers equation.
\end{abstract}

\section{Introduction}

\subsection{Thales and the origin of similarity}

If one adopts the point of view that one of the most fundamental laws of physics is that "a physical law establishes a relation between quantities having the same dimensions", and therefore these relations are independent of the system of units (one may simplify the units on both sides of the equation), one may affirm that science starts with Thales theorem relating the ratio of lengths in similar triangles (figure 1$)^{39},{ }^{36},{ }^{31}$. The Thales theorem is an example of similarity law, relating nondimensional quantities. The only parameter is the angle $\alpha$ at $\mathrm{A}$, between the straight lines, and hence a length $a$ must be related to other length $b$ by a relation of the type

$$
a=b f(\alpha),
$$

or as a relation between numbers $a / b=f(\alpha)$. This simple law permits sometimes to solve completely a problem, or more importantly to identify the appropriate parameters to describe a phenomenon. One example of a problem that can be solved using dimensional analysis, the theory generalizing the Thales statement, is the Pythagoras relation between the 


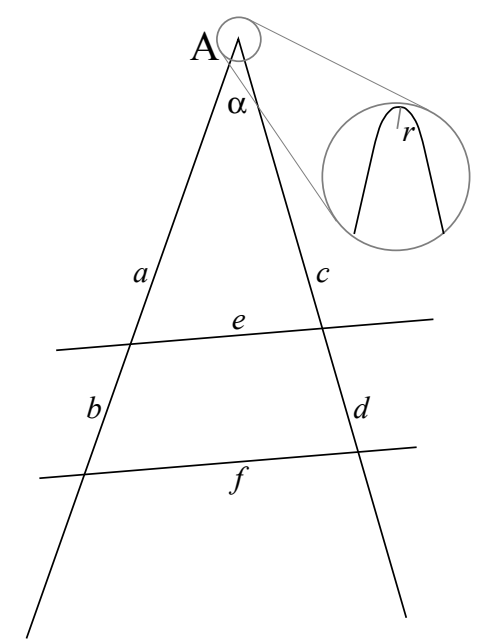

Figure 1. Thales theorem is a first scientific example of a rigorous similarity law relating lengths $a, b, \cdots$, through some unknown function of the angle $\alpha$. The Thales construction takes the curvature radius $r$ at A strictly equal to zero.

hypothenuse and the sides of a rectangular triangle. Using figure 2 one can say that the area of $\mathrm{ABC}$ is $S=c^{2} f(\alpha)$ (defining the hypothenuse $c$ as the length unit) and the areas of the two triangles ABF (hypothenuse $a$ ) and BCF (hypothenuse $b$ ) are $S_{1}=a^{2} f(\alpha)$ and $S_{2}=b^{2} f(\alpha)$ respectively, where $f(\alpha)$ is some function of the angle. Combining these expressions in $S=S_{1}+S_{2}$, one obtains Pythagoras theorem $c^{2}=a^{2}+b^{2}$.

One important point that can already be mentioned in the framework of the Thales theorem is the relation between self-similarity and the absence of intrinsic scale. Imagine that the vertex A is in fact round, with curvature radius $r$, as shown in figure 1 . In such a case Thales theorem does not apply and we have instead a relation of the more general form

$$
a / b=g(\alpha, r / b)
$$

In this case Thales theorem is recovered in the limit $r \rightarrow 0$, if the limit $g(\alpha, r / b) \rightarrow g(\alpha, 0)=f(\alpha)$ exists and does not vanish. Therefore, we find that the self-similar law is recovered when the intrinsic length $r$ disappears (or as usual in physics, can be neglected with respect to other lengths in the system, here $r \ll b, b$ being the length unit in this example). 


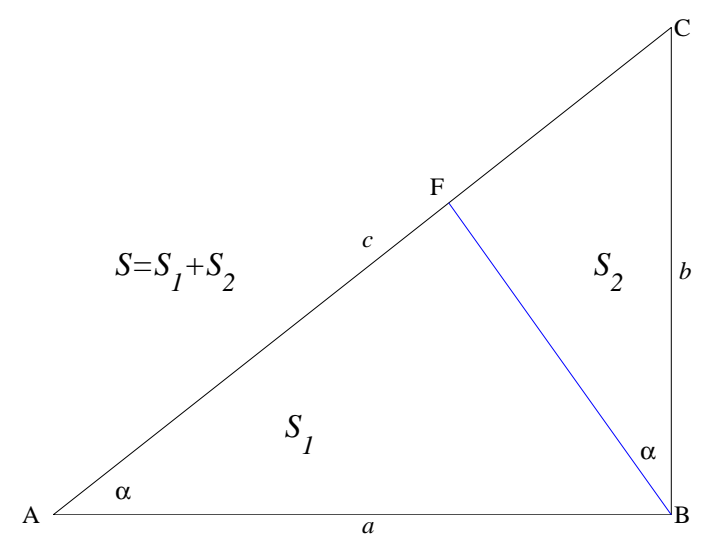

Figure 2. Demonstration of the Pythagoras theorem using dimensional analysis.

\subsection{The selection of relevant parameters}

The ideas of self-similarity play a central role in physics, they are related to the scaling transformations, and as other symmetries, they reflect important and deep properties of a system. However, self-similarity has a particular status, different to other symmetries, as for instance continuous symmetries that give rise to conservation laws, in the sense that most applications of self-similarity are as intermediate asymptotics, and not as some exact property of the system ${ }^{2}$. The fact that self-similarity applies in some region of scales is at the heart of its universality. This means that for systems having a wide variety of scales, one can isolate a range of values between some inner region ("microscopic" scale) and some outer region ("macroscopic" scale), in between of which the system behaves almost scale invariant. One simple example can illustrate this statement. When considering the oscillation period of a pendulum of length $\ell$, one obtains by dimensional analysis that $T=\sqrt{\ell / g} f(\theta)$, where $\theta$ is the (initial) angle with respect to the vertical, and $g$ the acceleration of gravity. One can suppose that $f(\theta)$ is a number of order one. However, this formula assumes implicitly that the initial velocity $v_{0}$ of the pendulum does not influence the period. If we add $v_{0}$ to the list of dimensional parameters, we obtain $T=\sqrt{\ell / g} F\left(\theta, v_{0} / \sqrt{g \ell}\right)$, with the additional nondimensional parameter $\Pi=v_{0} / \sqrt{g \ell}$. It is customary to denote dimensionless quantities by a capital $\Pi$, in accordance with the "П-theorem" of dimensional analysis ${ }^{35}$. That the period is given by $T=\sqrt{\ell / g}$, supposes thus the limit $F \rightarrow f$ for $\Pi \ll 1$. This is effectively the case as can be demonstrated by the complete solution of the problem and 
taken the limit of small oscillations; one knows, however, that for finite $\Pi$ nonlinear corrections of the pendulum period (depending on the oscillation amplitude, $\Pi$ ) appear. Therefore, the simple formula $T \approx \sqrt{\ell / g}$, is valid for a limited region of parameters in the limit $\Pi \rightarrow 0$.

Obviously, many other physical effects can enter into the problem, the difficulty is to determine the minimum number of relevant parameters. For instance, in the present problem we are considering a particle moving in a region much more smaller than the earth dimensions, and assume then that the gravitational field can be approximated by a constant: $\theta \ell \ll R_{\oplus}$, with $R_{\oplus}$ the earth's radius. We are also considering that the variations $\Delta \ell \approx m g / k$ in the length $\ell$ related to the elasticity of the wire are negligible (here we put $m$ the mass of the pendulum and $k$ the elastic constant of the wire), and that the size $d$ of the sphere $m$ is small enough to air friction be neglected during one period $d \ll(m / 6 \pi \mu) \sqrt{\theta g / \ell}$ ( $\mu$ is the air viscosity). We note then that the simple law for the period, even in the small oscillations limit, holds only in the region limited by $m g / k \ll \ell \ll(m / 6 \pi \mu d)^{2} g$, and a fortiori $\ell \ll R_{\oplus}$. This analysis show how one can discard in principle, a number of dimensional parameters $R_{\oplus}, k, \mu$, etc., by implicitly assuming that the corresponding dimensionless parameter leads to a finite limit when neglected.

\subsection{Similarity solution of the diffusion equation}

A more interesting example is given by the diffusion equation,

$$
u_{t}=D u_{x x}
$$

in an infinite domain, where $u=u(x, t)$ is related to the concentration of the solute, and $D$ the diffusion coefficient (partial derivatives are denoted by subscripts). Putting

$$
u(x, 0)=Q \frac{\mathrm{e}^{-x^{2} / \ell^{2}}}{\sqrt{\pi \ell^{2}}},
$$

with $Q$ and $\ell$ constants characterizing the amplitude and size of the initial concentration distribution, the solution of (1) reads,

$$
u(x, t)=Q \frac{\mathrm{e}^{-x^{2} /\left(\ell^{2}+4 D t\right)}}{\sqrt{\pi\left(\ell^{2}+4 D t\right)}} .
$$

Alternatively, one can try to obtain a solution of the diffusion equation in the limit of long times. Having a length unit, $\ell$, and a diffusion coefficient, $[D]=\mathrm{L}^{2} / \mathrm{T}$ (we use $\mathrm{L}, \mathrm{T}$ and $\mathrm{M}$ as the dimensions of length, time and mass, 
respectively), it is easy to form a diffusion time $\ell^{2} / 4 D$. Then "long times" means times much larger than this diffusion time, for which the solution $u$ can be thought to be independent of $\ell$. In such a case the method of dimensional analysis gives us a general framework to find the form of $u$. Taking into account that the only dimensional parameters of the problem are $[D]=\mathrm{L}^{2} \mathrm{~T}^{-1}$ and $[Q]=\mathrm{UL}$, where we called $\mathrm{U}$ the units of $u$, together with the variables $[x]=\mathrm{L}$ and $[t]=\mathrm{T}$, only two dimensionless quantities can be formed:

$$
\Pi_{1}=\frac{x}{\sqrt{D t}}, \text { and } \Pi_{2}=\frac{\sqrt{D t} u}{Q},
$$

implying the relationship,

$$
\Pi_{2}=\operatorname{func}\left(\Pi_{1}\right)
$$

(we denote by func $(\cdot)$ an arbitrary function of its arguments). Equation (3) is an example of similarity solution, that is a relation between dimensionless parameters, where the invariance with respect to a change in the units of measure, leads to a reduction in the number of the original independent variables. Because of the mathematical structure of the physical units, that are expressed as monomial functions, the similarity solution is generally in the form of a power law.

Hence, for times much longer than the diffusion time, one can try a similarity solution of (1) in a form like (3):

$$
u(x, t)=A \frac{\Phi(X)}{\sqrt{4 D t}}, \quad X=x / \sqrt{4 D t},
$$

where $A$ is a dimensional constant, $X$ is the independent nondimensional variable, and $\Phi$ the nondimensional diffusion field. Inserting (4) into (1), and noting that $\Phi$ satisfies an ordinary differential equation $\Phi+X \Phi_{X}=$ $(X \Phi)_{X}=-2 \Phi_{X X}$, one obtains the function

$$
\Phi(X)=\frac{\mathrm{e}^{-X^{2}}}{\sqrt{\pi}},
$$

where the normalization is such that $\int d X \Phi=1$. In terms of the original variables,

$$
u(x, t)=A \frac{\mathrm{e}^{-x^{2} / 4 D t}}{\sqrt{4 \pi D t}} .
$$


The arbitrary constant $A$, can be related to the initial condition: one can put

$$
\int_{-\infty}^{\infty} d x u(x, t)=Q
$$

because this integral is independent of time, with $u=u(x, 0)$. Therefore, when $t \gg \ell^{2} / 4 D$ we retrieve the similarity solution(2), provided that $Q=$ $A=\int d x u(x, 0)$. This example shows that the similarity solution (5) is in fact an asymptotic solution, valid for long enough times, irrespectively of the details of the initial condition: for long enough times the dependency of the solution on the characteristic length $\ell$, related to the shape of the initial solute distribution, disappears.

Another more interesting method, alternative to the method of dimensional analysis, to obtain similarity solutions of the kind of (4), is by the use of a scaling transformation,

$$
x \rightarrow x=a x^{\prime}, t \rightarrow t=b t^{\prime}, x \rightarrow u=c u^{\prime} .
$$

When this transformation is inserted into Eq. (1), it gives $u_{t^{\prime}}^{\prime}=u_{x^{\prime} x^{\prime}}^{\prime}$ and $\int u^{\prime} d x^{\prime}=1$, provided that the constants $a, b$ and $c$ satisfy

$$
a=\sqrt{D b}, c=Q / \sqrt{D b} .
$$

Further application of a similar scaling transformation would left the form of the equations unchanged, showing that (1) is invariant with respect to the one-parameter $b$ family transformation. This means that $x$ and $t$ are in fact not independent variables, but for instance, when $t$ is multiplied by $2, x$ is multiplied by $\sqrt{2}$, and simultaneously the amplitude is rescaled by a factor $\sqrt{2}$ : the graph of the function is the same at any time, if the $x$ and the $u$ axes are conveniently rescaled (this is the property allowing the expression of "similarity" or "self-similar" solution). The existence of such a similarity relationship allows us to choose $b$ in a convenient form, in order to reduce the number of variables: $b=t$. With this choice we find,

$$
u=c u^{\prime}\left(x^{\prime}, t^{\prime}\right)=c u^{\prime}(x / a, t / b)=(Q / \sqrt{D t}) u^{\prime}(x / \sqrt{D t}, 1),
$$

which is of the form (4).

The methods of similarity applied to variety of physical problems are extensively treated in the literature, we just mention some relevant references: the classical book by Sedov ${ }^{35}$, the book by Ames ${ }^{1}$ containing some interesting mathematical issues, and the important book by Barenblatt ${ }^{2}$, where the author thoroughly discusses the difficult subject of second kind 
similarity; the reader can also consult with some profit the review by Gratton ${ }^{20}$, and the paragraph on implosion in the Landau and Lifshitz book on fluid mechanics ${ }^{29}$. A deep relation exists between self-similarity and the renormalization group, this relation is investigated and clearly exposed in the book by Goldenfeld ${ }^{18}$.

\section{Free surface problems in the lubrication approximation}

\subsection{Thin film equations}

We consider the flow of a viscous thin film, driven by gravity and surface tension. We assume that the typical length scale of the free surface deformation $\ell$ is much larger than the film depth $L$. Under such conditions one can introduce the so called lubrication approximation of the Navier-Stokes equations, in order to obtain a simplified description of the interface $h(x, t)$ motion ${ }^{8},{ }^{32}$. We refer to figure 3 for the definition of the geometry and the notation.

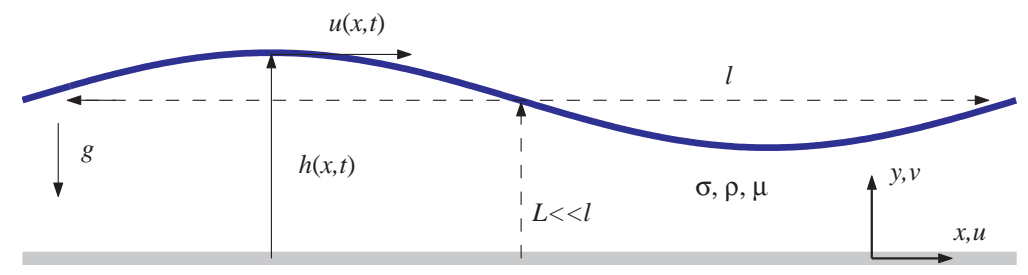

Figure 3. A thin liquid liquid film. The interface is described by the function $h(x, t)$ and the velocity $u(x, t) ; \sigma$ is the surface tension, $\rho$ the density, and $\mu$ the viscosity; $g$ is the acceleration of gravity; $L$ is the typical depth and $l$ the typical deformation length of the interface; we assume $L \ll l$. In two dimensions, coordinates are $(x, y)$, and the bulk velocity is $(u, v)=\mathbf{v}(x, y, t)$.

The Navier-Stokes equations of a thin layer of liquid over a solid substrate, in two dimensions, are

$$
\left\{\begin{array}{c}
u_{x}+v_{y}=0, \\
\rho\left(u_{t}+u u_{x}+v u_{y}\right)=-p_{x}+\mu\left(u_{x x}+u_{y y}\right), \\
\rho\left(v_{t}+u v_{x}+v v_{y}\right)=-p_{y}-\rho g+\mu\left(v_{x x}+v_{y y}\right),
\end{array}\right.
$$

These equations are completed with the kinematic boundary conditions

$$
\left\{\begin{array}{l}
u=v=0, \text { at } y=0, \\
h_{t}+u h_{x}=v, \text { at } y=h(x, t),
\end{array}\right.
$$


and the dynamical conditions at the free surface $y=h(x, t)$,

$$
-p \hat{\mathbf{n}}+S \cdot \hat{\mathbf{n}}=\sigma \kappa \hat{\mathbf{n}}, S=\mu\left(\begin{array}{cc}
2 u_{x} & u_{y}+v_{x} \\
u_{y}+v_{x} & 2 v_{y}
\end{array}\right)
$$

where $\kappa=-\nabla \cdot \hat{\mathbf{n}}$ is the interface curvature, $\hat{\mathbf{n}}=\left(-h_{x}, 1\right) / n$ is the unit normal vector at $(x, h(x, t))$, with $n=\left(1+h_{x}^{2}\right)^{1 / 2}$, and $S$ is the stress tensor. The normal component $\hat{\mathbf{n}} \cdot S \cdot \hat{\mathbf{n}}$ of (8) gives,

$$
-p+\frac{2 \mu}{n^{2}}\left[v_{y}\left(1-h_{x}^{2}\right)-h_{x}\left(u_{y}+v_{x}\right)\right]=\sigma \frac{\partial}{\partial x} \frac{h_{x}}{\left(1+h_{x}^{2}\right)^{1 / 2}},
$$

when $\mu=0$ this equation reduces to the Laplace formula relating the pressure jump at the interface between two fluids due to surface tension. The tangential component $\hat{\mathbf{t}} \cdot S \cdot \hat{\mathbf{n}}$ of $(8)$, with $\hat{\mathbf{t}}=\left(1, h_{x}\right) / n$ the unit tangent vector to the interface, gives,

$$
\left(u_{y}+v_{x}\right)\left(1-h_{x}^{2}\right)-4 h_{x} u_{x}=0 .
$$

The set of equations (6) with the boundary conditions (7-9-10) form a complete system describing the dynamics of the liquid film. It can be greatly simplified for slow and viscous flow in the limit of large wavelength $L \ll \ell$.

Indeed, let us assume that inertia is negligible with respect to viscous terms, and, in accordance with the geometrical condition $L \ll \ell$, that gradients in the vertical direction are sharper than in the horizontal direction $\left(u_{x} \ll u_{y}\right)$. In such a case, the $x$ component of the Navier-Stokes equation (6) would reduce to

$$
0=-p_{x}+\mu u_{y y} .
$$

Assume in addition, that in the vertical direction the fluid is essentially in hydrostatic equilibrium,

$$
0=-p_{y}-\rho g .
$$

Using the same approximation one would neglect $h_{x}$ with respect to 1 , and write the boundary conditions in the simple form:

$$
h_{t}+u h_{x}=v, u_{y}=0, p=-\sigma h_{x x},
$$

at $y=h$, and $u=v=0$ at $y=0$, where we suppose capillary effects of the same order as gravitational ones. These qualitative considerations can be put in a more rigorous level by introducing the small parameter $\epsilon=L / \ell$, together with the nondimensional parameters: $\operatorname{Re}=\rho U_{0} L / \mu=\epsilon \rho U_{0} \ell / \mu$ the Reynolds number based on the characteristic velocity $U_{0}$ and the mean 
depth of the film $L$, it would tend to zero for $\epsilon \rightarrow 0 ; \mathrm{Ca}=\mu U_{0} / \epsilon^{3} \sigma$ the capillary number, that compares the viscous and capillary terms, and that we scaled conveniently in $\epsilon^{-3}$ to keep it of the same order as the pressure; and $\mathrm{Gr}=\rho g L^{2} \epsilon / \mu U_{0}$ the "gravity" number, a ratio between gravity and viscous effects, also conveniently scaled to make it of the same order as the pressure term in the vertical component of the Navier-Stokes equation. In order to make these choices clear, we introduce the dimensionless variables,

$$
X=x / \ell, Y=y / L=y / \epsilon \ell, T=\left(U_{0} / \ell\right) t, U=u / U_{0}, V=v / \epsilon U_{0},
$$

(note the different scalings in the $x$ and $y$ directions) and replace they in the continuity equation:

$$
U_{X}+V_{Y}=0
$$

showing that the scaling of $v$ ensures that these two terms are of the same order, and in the $x$-component of the motion equation:

$$
\rho \frac{U_{0}^{2}}{\ell}\left(U_{T}+U U_{X}+V U_{Y}\right)=-\frac{1}{\ell} p_{X}+\frac{\mu U_{0}}{\epsilon^{2} \ell^{2}}\left(\epsilon^{2} U_{X X}+U_{Y Y}\right),
$$

or equivalently

$$
\epsilon \operatorname{Re}\left(U_{T}+U U_{X}+V U_{Y}\right)=-P_{X}+\epsilon^{2} U_{X X}+U_{Y Y},
$$

where

$$
P=\left(\epsilon \ell / U_{0} \mu\right) p
$$

gives the convenient scaling of the pressure: when $\epsilon \rightarrow 0$ we obtain $-P_{X}+$ $U_{Y Y}=0$, the nondimensional version of (11). Using now Eqs. (14) and (15) in the vertical component of the Navier-Stokes equation, we obtain

$$
\epsilon^{3} \operatorname{Re}\left(V_{T}+U V_{X}+V V_{Y}\right)=-P_{Y}-\mathrm{Gr}+\epsilon^{2} V_{Y Y}+\epsilon^{4} V_{X X}
$$

which reduces to (12) for vanishing $\epsilon$ (this justifies the choice of Gr). Obviously, one can also consider the case $g=0$, in which the gravitational effects are negligible. This does not change the scaling in the pressure and we simply would obtain $P_{Y}=0$ : the pressure is determined by the capillary boundary condition at the interface.

One easily verifies that the boundary conditions become

$$
\begin{aligned}
& H_{T}+U H_{X}=V, \\
& U_{Y}=4 \epsilon^{2}\left[H_{X} U_{X} /\left(1-\epsilon^{2} H_{X}^{2}\right)-V_{X}\right], \\
& -P+\mathrm{Ca}^{-1}\left(H_{X} / n^{1 / 2}\right)_{X}=\left(2 \epsilon^{2} / n^{2}\right)\left[V_{Y}\left(1-\epsilon^{2} H_{X}^{2}\right)-H_{X}\left(U_{X}+\epsilon^{2} V_{X}\right)\right],
\end{aligned}
$$


where $n^{2}=1+\epsilon^{2} H_{X}^{2}$, at $Y=H(X, T)$, and $U=V=0$ at $Y=0$. These conditions reduce to Eqs. (13) in the limit $\epsilon \rightarrow 0$. In summary, the lubrication approximation of the Navier-Stokes equations are:

$$
\left\{\begin{array}{l}
U_{X}+V_{Y}=0,-P_{X}+U_{Y Y}=0,-P_{Y}-\mathrm{Gr}=0, \\
H_{T}+U H_{X}=V, U_{Y}=0, P=-\mathrm{Ca}^{-1} H_{X X}, \text { at } Y=H(X, T), \\
U=V=0, \text { at } Y=0
\end{array}\right.
$$

These equations depend on two nondimensional parameters: $\mathrm{Gr}$ and $\mathrm{Ca}^{-1}$; Gr determines the contribution of hydrostatic effects to the pressure and $\mathrm{Ca}^{-1}$ determines the magnitude of the pressure jump due to surface tension. The condition that gravitational and capillary effects are of the same order $\mathrm{Gr} \sim \mathrm{Ca}^{-1}$, can be written as a condition on the characteristic length $\ell$. Indeed, assuming $\mathrm{CaGr}=\mathcal{O}(1)$ gives $\ell \sim a=(\sigma / \rho g)^{1 / 2}, a$ is the capillary length (independent to viscosity). Otherwise, further simplification is obtained by putting $\mathrm{Ca}^{-1}=0$ or $\mathrm{Gr}=0$, for the gravitational or capillary dominated regimes, respectively.

The system (16) can be reduced to a single equation for the interface $H(X, T)$, by direct integration. We first use the condition of hydrostatic equilibrium $P_{Y}=-\mathrm{Gr}=0$, to obtain the pressure $P$ as a function of $Y$ : $P(X, Y, T)-P_{0}(X, T)=-\mathrm{Gr} Y$, where we introduced the reference pressure $P_{0}$, independent of $Y$. Noting that $P_{X}=P_{0 X}$, the dependence of $U$ on the coordinate $Y$, is given by

$$
-P_{0 X}+U_{Y Y}=0, U(0)=0, U_{Z}(H)=0 \Rightarrow U=P_{0 X}\left(Y^{2} / 2-H Y\right) .
$$

In order to evaluate $P_{0}$ we use the normal-stress balance equation $P(H)=$ $-\mathrm{Ca}^{-1} H_{X X}$, from which we can write $P_{0}=P(H)+\mathrm{Gr} H$, or

$$
P_{0}=\mathrm{Gr} H-\mathrm{Ca}^{-1} H_{X X} .
$$

We integrate now the continuity equation between $Y=0$ and $Y=H(X, T)$, noting that $\int_{0}^{H} d Y V_{Y}=V(H)-V(0)$ and using $V(H)=H_{T}+U H_{X}$, we find

$$
H_{T}+U H_{X}+\int_{0}^{H(X, T)} d Y U_{X}=0 \Rightarrow H_{T}+\frac{\partial}{\partial X} \int_{0}^{H(X, T)} d Y U=0,
$$

where we applied the formula for the derivation of a definite integral: $(d / d x) \int_{0}^{h(x)} d y f(x, y)=\int_{0}^{h} d y f_{x}+h_{x} f$. Substituting $U$ into the last integral, and replacing the expression for the pressure, we finally get the desired thin film equation ${ }^{8}$ :

$$
H_{T}=\frac{\mathrm{Gr}}{3}\left(H^{3} H_{X}\right)_{X}-\frac{\mathrm{Ca}^{-1}}{3}\left(H^{3} H_{X X X}\right)_{X} .
$$


We already discussed the relevant regime of this equation, when the characteristic length of the motion $\ell$ is of the order of the capillary length $a$. If $\ell \ll a$ gravity effects can be neglected and the first term in (17) can be dropped. In the opposite case, if $\ell \gg a$, the first term in (17) dominates, and the thin film equation reduces to a nonlinear diffusion equation.

One can easily generalize Eq. (17) for three dimensional flows, where the interface is the surface $z=h(x, y, t)$. It is enough to note that the exterior derivative is a divergence, coming from the continuity equation; the following derivative comes from the pressure gradient; and the double derivative in the capillary term comes from a laplacian (given the curvature). Therefore, we can write:

$$
H_{T}=\frac{\mathrm{Gr}}{3} \nabla \cdot\left(H^{3} \nabla H\right)-\frac{\mathrm{Ca}^{-1}}{3} \nabla \cdot\left(H^{3} \nabla \Delta H\right),
$$

where $\nabla$ is the two-dimensional gradient operator.

\subsection{Nonlinear diffusion and the spreading of a drop}

In the limit $\ell \gg a$ the interface dynamics is governed by gravity, and Eq. (18) reduces to,

$$
h_{t}=\frac{\rho g}{3 \mu r}\left(r h^{3} h_{r}\right)_{r},
$$

in dimensional variables, for the axisymmetric case $(z=h(r, t)$ as shown in figure 4). It is analogous to a nonlinear diffusion problem, with a diffusion coefficient proportional to the cube of the "concentration", $h^{3}$. Equations of the form

$$
u_{t}=\nabla \cdot(D(u) \nabla u)
$$

arise in a great diversity of physical systems ${ }^{1},{ }^{2}$. A classical system described by a nonlinear diffusion equation is the heat conduction, when the conductivity depends on the temperature, as for instance in a collisional plasma; in this case $u$ represents the plasma temperature and the conductivity is essentially due to radiation, giving the power law $D \sim T^{5 / 2} 37$. Another example is the fluid flow through a porous medium, where the mathematical description is based on Darcy law, relating the mean velocity to the pressure gradient:

$$
\mathbf{v}=-\frac{b^{2}}{12 \mu} \nabla p
$$


( $b$ is a length characteristic of the pore size, and $b^{2} / 12$ is the "permeability" of the medium) and the continuity equation is

$$
\nabla \cdot(\rho \mathbf{v})=-(\alpha \rho)_{t}
$$

where $\alpha$ is the "porosity" of the medium, it takes into account the volume occupied by the pores, such that $\alpha \rho$ gives the actual mass of fluid per unit volume ( $\alpha$ might be variable, for instance if the pore is deformed by the fluid). Therefore, $\mathbf{v}$ in Eq. (21) is determined by the mass flux $Q \hat{\mathbf{n}}$ through a section of unit area in the flow direction, of the porous medium: $\mathbf{v}=(Q / \rho) \hat{\mathbf{n}}$. The system (21) and (22) is closed with the equation of state $p=$ func $(\rho)$, which for instance in the case of a gas is of the form $p / p_{0}=\left(\rho / \rho_{0}\right)^{\gamma}$, with $\gamma$ the ratio of specific heats $\left(\rho_{0}, p_{0}\right.$ are density and pressure reference constants, respectively). Using the equation of state in (21) and replacing in (22) on obtains a nonlinear diffusion equation with $D \propto \rho^{\gamma}$.

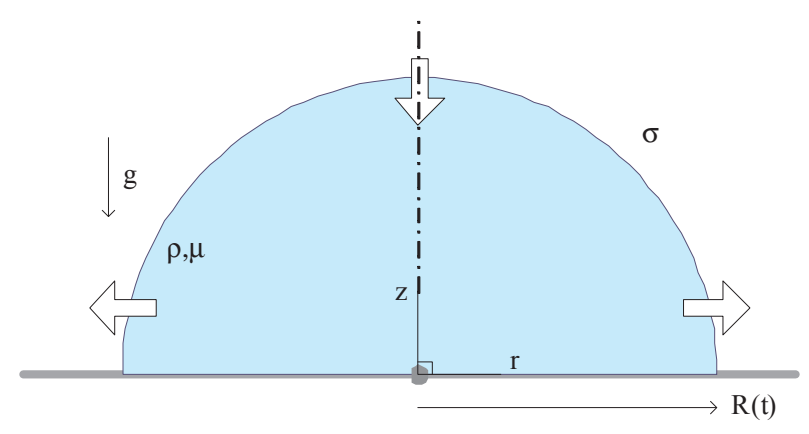

Figure 4. The spreading flow of a drop; the axisymmetric shape of the drop is given by $z=h(r, t)$, and the edge moves according to the law $r=R(t)$. We distinguish between the capillary $\sigma / \rho g \gg R^{2}$ and the gravitational $\sigma / \rho g \ll R^{2}$ regimes.

An interesting application of Eq. (19) is to the study of the creeping flow of a drop, draining under the action of gravity. We want to compute the motion of the edge $r=R(t)$ (figure 4 defines the geometry of the problem). The family of self-similar solutions of (19) can be obtained using the scale transformation $x \rightarrow x=a x^{\prime}, t \rightarrow t=b t^{\prime}$, and $h \rightarrow h=c h^{\prime}$, as in the case of the heat equation, leading to the relations,

$$
c / b=k c^{4} / a^{2}, c a^{2}=Q / 2 \pi,
$$

where we introduced the notation $k=\rho g / 3 \mu$ and supposed that the integral 
constraint

$$
Q=2 \pi \int_{0}^{\infty} r d r h(r, t)
$$

is satisfied. Hence, one gets $a=\left[(Q / 2 \pi)^{3} k b\right]^{1 / 8}$, and $c=(Q / 2 \pi k b)^{1 / 4}$, taking $b=t$,

$$
h(r, t)=\left(\frac{Q}{2 \pi k t}\right)^{1 / 4} H(X), X=\frac{r}{\left[(Q / 2 \pi)^{3} k t\right]^{1 / 8}} .
$$

If instead of having the constraint (23), which fix the similarity exponents, the physical problem under consideration depends on a dimensional variable $q$ having the dimensions $[q]=\left[x / t^{\alpha}\right]$, a one-parameter family of self-similar solutions would be obtained. The power $\alpha$ is a constant depending on the initial or boundary conditions, but, more generally, it can be arbitrary, or even unknown, and be determined by a solvability condition, given some relevant asymptotic behavior of the system. General power law solutions are obtained from the invariance condition of the system under a one-parameter group transformation,

$$
x \rightarrow x=\nu^{a} x^{\prime}, t \rightarrow t=\nu t^{\prime}, h \rightarrow h=\nu^{c} h^{\prime},
$$

where $\nu$ is the group parameter, and $a$ and $c$ powers to be determined (we choose the exponent of the $t$ scale factor equal to one). Upon substitution into (19) of the scaling transformation, the condition $c-1=4 c-2 a$, or $c=(2 a-1) / 3$, guarantees that the $\nu$ factors can be eliminated. This means that the ratios $h / t^{c}$ and $x / t^{a}$ are invariant under the scale transformation:

$$
h(r, t)=t^{(2 a-1) / 3} \operatorname{func}\left(x / t^{a}\right) .
$$

In order to simplify this equation we use the dimensional parameter $q$ together with $k=\rho g / 3 \mu$, to introduce the dimensionless variables $X=x / q t^{\alpha}$ (we must have $a=\alpha$ ), and $H=h(r, t) /\left(q^{2} / k\right)^{1 / 3} t^{(2 \alpha-1) / 3}$ :

$$
h(r, t)=\left(\frac{q^{2}}{k t^{1-2 \alpha}}\right)^{1 / 3} H(X), X=x / q t^{\alpha} .
$$

(We note that under the change of variables $h \rightarrow h=u / k^{1 / 3}$ the equation for $u$ does not contain dimensional parameters: $u_{t}=(1 / r)\left(r u^{3} u_{r}\right)_{r}$.) Returning to the constraint (23), we see that the integral $\int r d r h=Q / 2 \pi$, is independent of time if $(2 \alpha-1) / 3+2 \alpha=0$, thence $\alpha=1 / 8$ and $q=\left[(Q / 2 \pi)^{3} k\right]^{1 / 8}$, which are the results of $(24)$. 
Once the self-similar form (24) of the solution of (19) obtained, it is an easy task to find the ordinary differential equation satisfied by $H=H(X)$. Compute first the derivatives of $h$ :

$$
h_{t}=-\frac{1}{4 t}\left(\frac{Q}{2 \pi k t}\right)^{1 / 4} H-\frac{1}{8 t}\left(\frac{Q}{2 \pi k t}\right)^{1 / 4} H_{X} X
$$

and

$$
h_{r}=\left(\frac{Q}{2 \pi k t}\right)^{1 / 4} \frac{H_{X}}{\left[(Q / 2 \pi)^{3} k t\right]^{1 / 8}} .
$$

Then, derivatives with respect to $r$ are computed by the substitution rule:

$$
\frac{\partial^{n}}{\partial r^{n}}=\frac{1}{\left[(Q / 2 \pi)^{3} k t\right]^{n / 8}} \frac{\partial^{n}}{\partial X^{n}}
$$

Using these formulas, we realize that all dimensional and time dependent terms disappear, and obtain the nonlinear differential equation:

$$
-X H / 4-X^{2} H_{X} / 8=\left(X H^{3} H_{X}\right)_{X} .
$$

The left hand side is a total derivative $X H / 4+X^{2} H_{X} / 8=(1 / 8)\left(X^{2} H\right)_{X}$, allowing us to integrate (26) once, to get $-X H / 8=H^{3} H_{X}$ (the integration constant vanishes, by symmetry, at $X=0, H_{X}=0$, moreover we have to satisfy $H \rightarrow 0$ for $X \rightarrow \infty$, in such a way that $\int X d X H$ converges). Direct integration of $-X / 8=H^{2} H_{X}$ leads to,

$$
H(X)=\left(\frac{3}{16}\right)^{1 / 3}\left(X_{0}^{2}-X^{2}\right)^{1 / 3}
$$

where $X_{0}$ is the integration constant, which corresponds to the condition $H\left(X_{0}\right)=0$, then giving the edge position $X=X_{0}$, or in the dimensional variables,

$$
R(t)=X_{0}\left[(Q / 2 \pi)^{3} k t\right]^{1 / 8} .
$$

The shape (27) of the drop is shown in figure 5. The actual value of $X_{0}$ is determined by the integral constraint:

$1=\int_{0}^{X_{0}} X d X H(X)=\left(\frac{3}{16}\right)^{1 / 3} X_{0}^{8 / 3} \int_{0}^{1} y d y\left(1-y^{2}\right)^{1 / 3}=\frac{3}{8}\left(\frac{3}{16}\right)^{1 / 3} X_{0}^{8 / 3}$,

which gives the numerical result

$$
X_{0}=\left(\frac{8}{3}\right)^{3 / 8}\left(\frac{16}{3}\right)^{1 / 8} \approx 1.78
$$




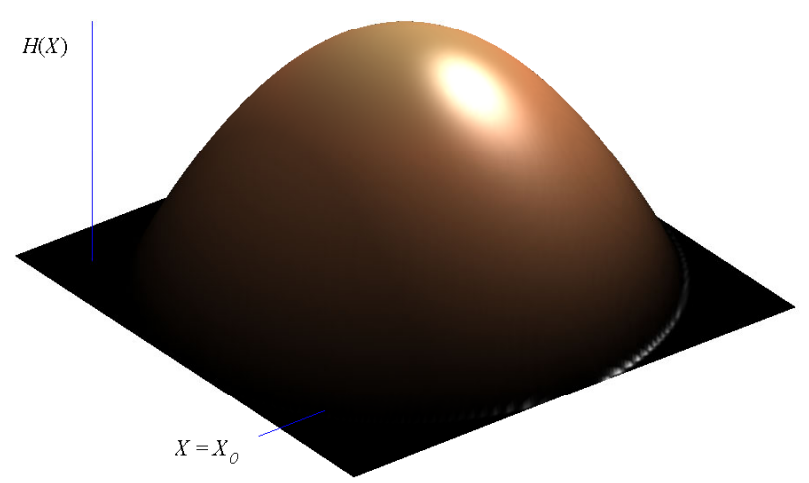

Figure 5. Shape of the drop in the gravitational regime, the position of the edge is given by the self-similar variable $X=X_{0}$, giving a power law $R \sim t^{1 / 8}$.

completing the solution of the creeping flow equation for the gravitational spreading of a viscous drop.

There is an extensive literature on the self-similar solutions of (19), see for example the works by Gratton et al. ${ }^{21},{ }^{10},{ }^{22}$, where cases of second kind similarity, arising for convergent currents, are also treated.

\subsection{The capillary regime of drop spreading}

The capillary regime of spreading is described by Eq. (17),

$$
-h_{t}=\frac{\sigma}{3 \mu}\left(h^{3} h_{x x x}\right)_{x},
$$

where we retained the second term in the right hand side and restored dimensional variables. We consider situations where the mass of liquid is constant:

$$
\int_{-\infty}^{\infty} d x h(x, t)=Q
$$

(We discuss the one dimensional case, but similar considerations are valid for the axisymmetric case.) The capillary driving spreading is by far a more complicated physical regime than the gravity dominated regime. The reason is that near the contact line, the edge of the drop where the liquid joins the solid and gas, (30) fails in giving a satisfactory physical solution. The energy integral associated to Eq. (30) is

$$
\mathcal{E}=\frac{\sigma}{2} \int_{-\infty}^{\infty} d x h_{x}^{2}
$$


proportional to $\int d x\left(1+h_{x}^{2}\right)^{1 / 2}$ the surface of the drop (per unit length, in the one dimensional case). The time evolution of the energy is easily computed using Eq. (30),

$$
\dot{\mathcal{E}}=-\frac{\sigma^{2}}{3 \mu} \int_{-\infty}^{\infty} d x h^{3} h_{x x x}^{2}=-3 \mu \int_{-\infty}^{\infty} d x \frac{u^{2}}{h}=-\mathcal{D},
$$

where we introduced the interface velocity,

$$
u=u(x, t)=(\sigma / 3 \mu) h^{2} h_{x x x} ;
$$

with this definition (30) takes the simple form $h_{t}+(h u)_{x}=0$ of a continuity equation. Equation (33) allows us to define a dissipation rate $-\dot{\mathcal{E}}=\mathcal{D}$. The difficulty appears clearly in Eq. (33): in the neighborhood of the contact line $x=x_{0}$ and $h\left(x_{0}\right)=0$, we can put $h \sim \xi^{\alpha}$, for $x=x_{0}-\xi$, and the condition of finite interface velocity $u \sim h^{3} h_{x x x} \sim \xi^{2 \alpha} \xi^{\alpha-3}$ gives $\alpha \geq 1$; under such conditions the dissipation integral

$$
\mathcal{D} \sim \int^{x_{0}-\xi} d x u^{2} / h \sim-\ln \xi
$$

( $\alpha=1$ for $u=$ const.) diverges logarithmically, and as a consequence Eq. (30) is incompatible with the spreading at constant velocity of a drop!. This is the well known problem associated to the moving contact line (see the reviews by de Gennes ${ }^{9}$, Oron ${ }^{32}$ and Pomeau ${ }^{33}$, and the papers in references $\left.{ }^{23},{ }^{11},{ }^{38},{ }^{17},{ }^{14}\right)$.

We can see an important difference with respect to the gravity case, in which the energy is of the form $\int d x h^{2}$, and the dissipation rate $\mathcal{D}=$ $\int h^{3} h_{x}^{2} \sim \int u^{2} / h$ with $u=-h^{2} h_{x}$; taking $h \sim \xi^{\alpha}$, and $u \sim \xi^{2 \alpha} \xi^{\alpha-1}$, we find $\alpha \geq 1 / 3$, the lower limit coinciding with the self-similar solution $\alpha=1 / 3$. The dissipation integral is convergent: $\mathcal{D} \sim-\int_{0} d \xi / \xi^{1 / 3}<\infty$. Therefore, the self-similar solution preserves the mass of the drop, and the dissipation rate is finite, thus giving an acceptable physical picture. This not the case for the capillary regime, for which solutions having finite contact angles ( $h_{x}$ at $x=x_{0}$ ), in order to move at a finite velocity, lead to diverging stresses. This divergence must be regularized by some additional physical process, that modifies the shape of the interface in the neighborhood of the contact line. The actual form of the regularization, no slip condition, phase transition at the contact line, van der Waals forces, are the object of debate. We limit ourselves to some general prescription by introducing an additional length, $b$, characteristic of the process in the vicinity of the 
contact line, where Eq. (30) breaks down:

$$
-h_{t}=\frac{\sigma}{3 \mu}\left[\left(h^{3}+b^{3-n} h^{n}\right) h_{x x x}\right]_{x},
$$

where the power $0<n<3$ depends on the model details. The problem is therefore to investigate self-similar solutions in the framework of intermediate asymptotics. Indeed, we cannot expect a simple power law in the form $x \sim t^{\alpha}$ to be valid for all times, in particular for long enough times the hight of the interface decreases, and will attain the small value $h \sim b$, for which the usual scaling fails. We expect thus that if some self-similar regime exists, it would be valid for lengths much larger than $h \gg b$ and times much shorter that the time for which $h(x, t) / b \sim 1$.

Let us apply first the usual method for the search of a similarity solution to the original Eq. (30). In order to simplify the notation we absorb the dimensional constants in the definition of variables, and note that (30) is invariant under the two-parameter scale transformation: $x \rightarrow x=a x^{\prime}$, $t \rightarrow t=\left(a^{4} / c^{3}\right) t^{\prime}, x \rightarrow h=c h^{\prime}$; taking also into account (31), the oneparameter family $x \rightarrow x=a x^{\prime}, t \rightarrow t=a^{7} t^{\prime}, x \rightarrow h=(1 / a) h^{\prime}$ remains, showing that the self-similar form of the solution is

$$
h(x, t)=\frac{1}{t^{1 / 7}} H(X), X=x / t^{1 / 7},
$$

(in the axisymmetric case the same reasoning gives $r \sim t^{1 / 10}$ ). The modified Eq. (34) possesses an additional dimensional parameter, thus $h(x, t)=$ $\left(1 / t^{1 / 7}\right) H(X, x / b)$, and the problem is to find the behavior of $H(X, x / b)$ for $b \rightarrow 0$. We introduce now the position of the contact line $x=R(t)$, the position for which $h=0$. The condition that $h \gg b$, means that $R \ll$ $Q / b$, from the $Q$ constraint (31); simultaneously the time condition derives from $h \sim b$, leading to $t \ll(3 \mu / \sigma)\left(Q^{4} / b^{7}\right)$. In summary, the intermediate asymptotics are

$$
\sqrt{Q} \ll R(t) \ll Q / b,(3 \mu / \sigma) \sqrt{Q} \ll t \ll(3 \mu / \sigma)\left(Q^{4} / b^{7}\right),
$$

where we estimated the length and time necessary to make the evolution independent to the initial condition. For this range of values we assume that $h$ can be written in the form

$$
h(x, t)=\frac{1}{R(t)} H\left(\frac{x}{R(t)}\right), \text { for } h \gg 1, R \ll 1,
$$

the approximate self-similar variable is $X=x / R(t)$ (therein we use the dimensionless expressions, with the choice $b=\mathcal{O}(1))$. The time scale is 
given by the rate $\dot{R} / R$, meaning that the velocity can be estimated by

$$
u \sim(\dot{R} / R) x .
$$

The energy is then given by

$$
-\dot{\mathcal{E}}=-\frac{1}{2} \frac{d}{d t} \int d x h_{x}^{2}=-\frac{1}{2} \frac{d}{d t} \frac{1}{R^{3}} \int d X H_{X}^{2} \sim \dot{R} / R^{4},
$$

and the dissipation rate,

$$
\mathcal{D}=2 \int_{0}^{x(h=1)} d x u^{2} / h
$$

where we cutoff the integral at the position for which $h=1$. To compute this position we develop $h(x)=h(R)+h_{x}(R)(x-R)=\left(H_{X} / R^{2}\right)(x-R)=1$, to obtain $x(h=1)=R(1-R / \theta)$, where $\theta=\left|H_{X}(R)\right|$ is the contact angle $(\theta \sim \mathcal{O}(1)$ is finite). Therefore, the above integral is logarithmically divergent in the limit $X \rightarrow 1$ (this is consistent with the assumption $R \ll$ 1 for the validity of the intermediate asymptotics). Using the change of variable $\xi=1-X$ and the development $H(X)=\theta(1-X)=\theta \xi$, the dissipation integral can be estimated as

$$
\mathcal{D}=2 R^{4}(\dot{R} / R)^{2} \int_{0}^{1-R / \theta} \frac{d X X^{2}}{H(X)} \sim-R^{2} \dot{R}^{2} \int^{R / \theta} \frac{d \xi}{\theta \xi} \sim R^{2} \dot{R}^{2} \ln \left(\frac{1}{R}\right),
$$

where we discarded the numerical and order one factors. The energy balance reduces to

$$
R^{6} \ln \left(\frac{1}{R}\right) \dot{R}=1
$$

which leads, after a simple manipulation, to the scaling factor:

$$
R(t)=\left[\frac{t}{\ln (1 / t)}\right]^{1 / 7},
$$

for $R \ll 1$ and $t \ll 1$, completing formula (36). Result (37) shows that the naive scaling $x \sim t^{1 / 7}$ acquires a logarithmic correction, whose physical origin can be traced back to the divergence of the dissipation rate at the contact line.

It is worth noticing that Eq. (37) is independent of $n$, or equivalently, of the microscopic model details (it depends however on the microscopic scale $b$ ). The spreading law (37) is valid until the film height is $h \approx 1$, for thinner film regions the dominant term in (34) becomes $b^{n} h^{3-n} \gg h^{3}$. The relevant equation in this region is thus $-h_{t}=\left(h^{3-n} h_{x x x}\right)_{x}$, and the usual 
dimensional analysis should hold (remember that $n<3$ ), leading to the self-similar solution,

$$
h(x, t)=\frac{1}{t^{1 /(4+n)}} H(X), X=x / t^{1 /(4+n)}, \text { for } h \lesssim 1, t \gg 1,
$$

equivalent to Eq. (35), but with the similarity exponent $1 /(4+n)$ instead of $1 / 7$.

\section{Second type similarity in a porous medium}

\subsection{The renormalization group}

We related the invariance of a physical system under a scale transformation to the existence of a self-similar evolution, at least in some asymptotic limit. The scaling group of transformation we used implied an arbitrary change in magnitudes, such as in the relation $x \rightarrow a x$, for which $x$ is changed by the arbitrary amount $a$. However, one may also consider the behavior of the system under infinitesimal changes in a continuous parameter.

Let us consider a system described by the function $x=x(t)$, and satisfying some differential equation $\dot{x}(t)=F(x(t))$. From the solution $x=X\left(t ; x_{0}, t_{0}\right)$ of this equation with the initial condition $x\left(t_{0}\right)=x_{0}$, we can generate a family of trajectories $x=X\left(t ; x_{0}\left(t_{0}\right), t_{0}\right)$ parameterized by $t_{0}$, which can be interpreted as the time at which we compute the initial condition $x\left(t_{0}\right)$. It is obvious that if we choose the values of $t_{0}$ and $x_{0}\left(t_{0}\right)$ in such a way that $X\left(t ; x_{0}\left(t_{0}\right), t_{0}\right)$ belongs to some particular trajectory $X\left(t ; x_{0}(0), 0\right)$, then the family collapses to this unique trajectory $X\left(t ; x_{0}(0), 0\right)=X\left(t ; x_{0}\left(t_{0}(t)\right), t_{0}(t)\right)$. Note that this is a kind of invariance condition on the trajectory $X$, with respect to changes in the value of $t_{0}$.

From a geometrical point of view, the condition for this invariance is that the envelope of the family coincides with the function itself. We recall that the envelope $x_{E}$ of a family of curves $F=X\left(t ; x_{0}\left(t_{0}\right), t_{0}\right)-x=0$ depending on a parameter $t_{0}$, is simply the curve satisfying simultaneously $F=0$ and $d F / d t_{0}=0$. These conditions allow to determine the function $t_{0}(t)$, which gives the envelope $X\left(t ; x_{0}(t), t_{0}(t)\right)-x_{E}=0$. Therefore, the envelope trajectory is determined by the solution of the equation,

$$
\frac{d}{d t_{0}} X\left(t ; x_{0}\left(t_{0}\right), t_{0}\right)=\frac{\partial X}{\partial t_{0}}+\frac{\partial X}{\partial x_{0}} \frac{d x_{0}}{d t_{0}}=0 \Rightarrow t_{0}=t_{0}(t) .
$$

thus giving $x_{E}=X\left(t ; x_{0}(t), t_{0}(t)\right)$. Equation (39) can be interpreted as being a "flow equation" of the initial condition "field" (to borrow the field theory jargon). The important point is that it establishes the invariance 
of the trajectory under a change in the reference time $t_{0}$ (invariance not to be confused with time translation symmetry!). However, in general the trajectory is not known a priori, and then it is impossible to solve (39). The interest of the method is that (39) can in fact be used to construct the trajectory from the knowledge of its perturbation expansion. This approach to the renormalization group was developed by Kunihiro ${ }^{26},{ }^{25},{ }^{15}$, as a natural development of the dynamical renormalization group originally devised by Chen, Ono and Goldenfeld ${ }^{19},{ }^{4},{ }^{5}$.

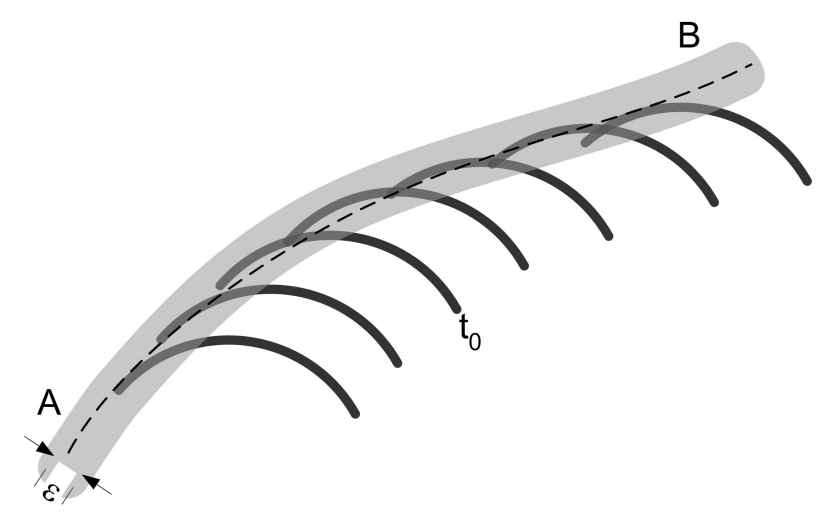

Figure 6. Scheme showing the actual AB trajectory of a system (dashed line) and its neighborhood of size $\epsilon$ (gray zone); the family of perturbation trajectories approach $\mathrm{AB}$ only near $t \approx t_{0}$ ( $t_{0}$ labels each member of the family). The envelope of these perturbation trajectories approach globally $\mathrm{AB}$ (within the tube $\epsilon$ ).

Indeed, if only an approximation of the trajectory is known $X_{\epsilon}$, computed from a perturbation expansion in a small parameter $\epsilon$, around $t=t_{0}$, the family of these trajectories are close to the actual exact one only for a time $t \sim \mathcal{O}(\epsilon)$ (see figure 6). Therefore, the envelope equation

$$
\frac{d}{d t} X_{\epsilon}\left(t ; x_{0}\left(t_{0}\right), t_{0}\right)=\left.\frac{\partial X_{\epsilon}}{\partial t_{0}}\right|_{t_{0}=t}+\left.\frac{\partial X_{\epsilon}}{\partial x_{0}} \frac{d x_{0}}{d t_{0}}\right|_{t_{0}=t}=0 \Rightarrow t_{0}=t_{0}(t, \epsilon),
$$

will give a global approximation $X=X_{\epsilon}\left(t ; t_{0}(t, \epsilon)\right)$ to the exact solution. Equations (39) or (40) are of the usual renormalization group form if one interprets $t_{0}$ as the logarithm of a scale parameter $t_{0}=\ln a$, hence $d X / d t_{0}=$ $a d X / d a$, gives the rate of change in $X$ following a change in scale $a$; if this rate vanishes, $X$ is scale invariant. In other words, if the system is described by the function $x(a)$ (we forget all other arguments) at scale $a$, and we change the scale by a factor $\lambda$, to get $x(\lambda a)$, the scale transformation $T$ 
allows to relate the system at two different scales: $x(\lambda a)=T_{\lambda} x(a)$; now let us assume that the change in scale is infinitesimal $\lambda=1+\epsilon$, we can write

$$
\frac{d x(a)}{d a}=\lim _{\epsilon \rightarrow 0} \frac{x(1+\epsilon a)-x(a)}{\epsilon a}=\left.\frac{1}{a} \frac{d T_{\lambda} x(a)}{d \lambda}\right|_{\lambda=1}=0,
$$

where the last equality is the condition of scale invariance, an equation having the same form as (39), once we realize that $T_{\lambda} X(a)$ is the prescription to compute the trajectory $x=X\left(t ; x_{0}\left(t_{0}\right), t_{0}\right)$.

One simple example might be the single function $x=X(a)$ satisfying $X(\lambda a)=\lambda^{\alpha} X(a)$, thus $x=\lambda^{-\alpha} X(\lambda a)=\lambda^{-\alpha} X(\xi)$, with $\xi=\lambda a$. Direct differentiation gives: $d \ln x / d \ln \lambda=\partial \ln x / \partial \ln \lambda+$ $(\partial \ln X / \partial \ln \xi)(d \ln \xi / d \ln \lambda)=0$, condition of scale invariance in which we recognize the "renormalization group" equation. Noting that $d \ln \xi / d \ln \lambda=$ 1 , and $\partial \ln x / \partial \ln \lambda=-\alpha$, this condition writes $-\alpha+\partial \ln X / \partial \ln \xi=0$, or after integration, $X(\xi)=\xi^{\alpha}=(\lambda a)^{\alpha}$, or $x=X(a)=a^{\alpha}$ (up to a multiplicative constant), that is the basic monomic function satisfying scaling invariance.

\subsection{The Barenblatt equation}

The renormalization group is usually applied in statistical physics as a "nonperturbative" method allowing the computation of "anomalous" exponents in phase transitions ${ }^{18},{ }^{45}$. In the framework of dynamical systems these anomalous exponents can be related to the departure of the system's scaling properties to the "normal" exponents given by dimensional analysis, the second kind similarity in the classification of Barenblatt ${ }^{2}$. In order to illustrate these ideas we study the flow in an elasto-plastic porous medium of a liquid, using a simple nonlinear diffusion equation. This medium is characterized by an irreversible deformation of the pores in response to the flow of the fluid through it. The flow is described by Darcy's equation (21) and the continuity equation (22), where the porosity (volume) is related to the pressure (stress) by a linear constitutive relation $\alpha=\alpha_{0}+\left(p-p_{0}\right) / \kappa$, with $\kappa$ a constant depending on the elastic properties of the medium (when $\kappa \rightarrow \infty$ the medium is rigid), and $p_{0}$ a reference fluid pressure. The plastic properties of the pores are taken into account through a variation of $\kappa$ with the pressure: $\kappa=\kappa_{1}$ if the fluid enters into the pore $p_{t}>0$, and $\kappa=\kappa_{0}<\kappa_{1}$ if the fluid leaves the pore $p_{t}<0$. Within the same order of approximation, the equation of state can be written as $\rho=\rho_{0}+\beta\left(p-p_{0}\right)$, which takes into account the weak compressibility $\beta$ of the medium. Replacing these relations, using the excess pressure $u=p_{0}-p$ as the new variable, and 
retaining only the linear terms in $u$, we arrive at:

$$
u_{t}=D \Delta u, D=\frac{b^{2} \rho}{12 \mu}\left\{\begin{array}{l}
\frac{\kappa_{0}}{\alpha_{0} \beta \kappa_{0}+\rho_{0}}, \text { for } u_{t}>0 \\
\frac{\kappa_{1}}{\alpha_{0} \beta \kappa_{1}+\rho_{0}}, \text { for } u_{t}<0
\end{array},\right.
$$

or after nondimensionalization,

$$
u_{t}=\frac{1}{2}\left[1+\epsilon \Theta\left(-u_{t}\right)\right] \Delta u
$$

where $\Theta$ is the Heaviside function; the small parameter $\epsilon$ is proportional to the difference $\kappa_{1}-\kappa_{0}$. Equation (42) is the Barenblatt equation for the diffusion in an elasto-plastic medium, the main difference with the usual diffusion equation is that the diffusion coefficient is a discontinuous function, although the pressure (the diffusing field in this case) is continuous and with continuous derivatives up to second order. As a consequence, the conservation law $(d / d t) \int d x u=0$ is no more valid, meaning that $Q=Q(t)$ becomes a function of time. Form the dimensional analysis point of view, Barenblatt equation has the same properties as the diffusion equation, and in principle one may expect a similarity solution of the type (4). However, due to the variation of $Q$ with time, one may suspect an influence of the actual value of $Q\left(t_{0}\right)$ at time $t_{0}$, and thus the contribution of an additional parameter $t_{0}$ related to the initial conditions, to the list of dimensional parameters of the problem:

$$
u=u\left(x, t ; t_{0}, \epsilon\right)=(Q / \sqrt{t}) U\left(x / \sqrt{t}, t_{0} / t, \epsilon\right) .
$$

In dimensional analysis the usual assumption is that $U \rightarrow$ const. $\neq 0$ for $t_{0} / t \rightarrow 0$, in such a way that for long times the system trajectory is independent of the scale $t_{0}$. Situations where this reasoning fails are well known in statistical physics. In the neighborhood of a phase transition fluctuations of all scale lengths are present, meaning that the lattice size (or some other length cutoff) will play a role, even if the system's size tends to infinity. The influence of the length cutoff reflects in the appearance of anomalous exponents. Mimicking these considerations, in our case we can infer that for $t_{0} / t \rightarrow 0$ the dimensionless excess pressure behaves anomalously, $U \rightarrow\left(t_{0} / t\right)^{\alpha}$,

$$
u=\frac{M}{t^{1 / 2+\alpha(\epsilon)}} U(X, \epsilon), X=\frac{x}{\sqrt{t}},
$$

where we note the $\epsilon$ dependence of the anomalous exponent, here $M$ is a constant of the form $M=Q\left(t_{0}\right) t_{0}^{\alpha}$. The power law dependence of $P$ 
explains the failure of dimensional analysis, which implicitly assumes that the asymptotic limit, when some scale becomes irrelevant, would be a simple (finite) constant.

\subsection{Perturbation expansion and renormalization group solution}

We apply the method described above, that starts with the computation of a perturbative solution, valid locally around some fixed time $t_{0}$, over which the renormalization group, based on the invariance of the system with respect to the "scale" $t_{0}$, allows to find a global solution. We use a slightly improved version of the method introduced by Kunihiro ${ }^{25}$, and Goldenfeld ${ }^{19}$, which do not need a renormalization step to eliminate divergences in the perturbation series. Therefore, we try to perturbatively solve the Barenblatt equation using an expansion in powers of $\epsilon$. When $\epsilon$ vanishes the equation reduces to the diffusion equation, whose general solution can be given in terms of the Green function,

$$
G(x, t)=\frac{\mathrm{e}^{-x^{2} / 2 t}}{\sqrt{2 \pi t}},
$$

allowing to formally write the solution of (42), as

$$
\begin{aligned}
u(x, t)= & \int_{-\infty}^{\infty} d y G\left(x-y, t-t_{0}\right) u\left(y, t_{0}\right)+ \\
& \frac{\epsilon}{2} \int_{t_{0}}^{t} d s \int_{-\infty}^{\infty} d y G(x-y, t-s) \Theta\left(-u_{s}(y, s)\right) u_{y y}(y, s) .
\end{aligned}
$$

We develop $u$ in powers of $\epsilon$ to obtain the expansion $u=u^{(0)}+\epsilon u^{(1)}$. At zero order, the solution of the diffusion type equation is the Gaussian (cf. Eq. (5)),

$$
u^{(0)}\left(x, t ; t_{0}\right)=Q\left(t_{0}\right) \frac{\mathrm{e}^{-x^{2} / 2 t}}{\sqrt{2 \pi t}}, u^{(0)}\left(x, t_{0} ; t_{0}\right)=Q\left(t_{0}\right) \frac{\mathrm{e}^{-x^{2} / 2 t_{0}}}{\sqrt{2 \pi t_{0}}},
$$

where we explicitly take into account the dependence of $Q$ on the choice of the initial time $t_{0}$. The first order equation, relating $u^{(1)}$ to $u^{(0)}$, can be written as,

$$
u^{(1)}=\frac{1}{2} \int_{t_{0}}^{t} \frac{d s}{\sqrt{2 \pi(t-s)}} \int_{-\infty}^{\infty} d y \exp \left[-\frac{(x-y)^{2}}{2(t-s)}\right] \Theta\left(-u_{s}^{(0)}\right) u_{y y}^{(0)},
$$

where we used the Green function (44). Using the zero order result, and introducing the position $x_{0}(t)$ where $u_{t}^{(0)}=0$ (we do not need to know this 
position at higher orders in $\epsilon$ ), we compute

$$
\Theta\left(-u_{s}^{(0)}\right) u_{y y}^{(0)}=Q\left(\frac{y^{2}}{s}-1\right) \frac{\mathrm{e}^{-y^{2} / 2 s}}{s \sqrt{2 \pi s}} \Theta\left(x_{0}(s)-|y|\right),
$$

and substitute it into Eq. (46), to obtain after the change of variable $Y=$ $y / \sqrt{s}$,

$$
u^{(1)}=\frac{Q}{4 \pi} \int_{t_{0}}^{t} \frac{d s}{s \sqrt{(t-s)}} \int_{-1}^{1} d Y \mathrm{e}^{-Y^{2} / 2}\left(Y^{2}-1\right) \exp \left[-\frac{(x-Y \sqrt{s})^{2}}{2(t-s)}\right],
$$

where we have taken into account that $x_{0}(s)= \pm \sqrt{s}$, or $Y= \pm 1$ in the new variable (to this order in $\epsilon$ ). We remark in the above expression that logarithmic terms will arise due to the pole at $s=0$. We then try to isolate the contribution to the integral of this term with respect to the other secular terms (proportional to $t-t_{0}$ in the perturbation series). The logarithmic term is the dominant one in limiting the validity region of the expansion in the vicinity of $t_{0}$, expressing the fact that initially $u^{(0)}$ is, in same sense, infinitely far from the actual initial state. The role of the renormalization group is to remove this divergence, using the scaling properties of the system at some finite distance from the initial state. The most straightforward way to extract the logarithmic term is to see what happens for $s=0$. The dominant part of the integral around $s=0$ is

$$
u^{(11)}=\frac{Q}{2 \sqrt{2 \pi}} \frac{\mathrm{e}^{-x^{2} / 2 t}}{\sqrt{2 \pi t}} \int_{t_{0}}^{t} \frac{d s}{s} \int_{-1}^{1} d Y \mathrm{e}^{-Y^{2} / 2}\left(Y^{2}-1\right),
$$

the remaining part is,

$$
\begin{aligned}
u^{(12)}=\frac{Q}{4 \pi} \int_{t_{0}}^{t} \frac{d s}{s} \int_{-1}^{1} d Y & \mathrm{e}^{-Y^{2} / 2}\left(Y^{2}-1\right) \times \\
& {\left[\frac{\mathrm{e}^{-(x-Y \sqrt{s})^{2} / 2(t-s)}}{\sqrt{(t-s)}}-\frac{\mathrm{e}^{-x^{2} / 2 t}}{\sqrt{t}}\right] . }
\end{aligned}
$$

Therefore, we write $u^{(1)}=u^{(11)}+u^{(12)}$, where the first term gives, after performing the integral, a logarithmic contribution

$$
u^{(11)}=-\frac{Q \mathrm{e}^{-x^{2} / 2 t}}{\sqrt{2 \pi t}} \frac{1}{\sqrt{2 \pi \mathrm{e}}} \ln \frac{t}{t_{0}},
$$

and the second term, when developed around $t=t_{0}$, reveals secular terms in powers of $\left(t-t_{0}\right) / t$,

$u^{(12)} \approx-\frac{Q \mathrm{e}^{-x^{2} / 2 t}}{\sqrt{2 \pi t}}\left[\operatorname{erf}\left(\frac{1}{\sqrt{2}}\right)-\frac{2}{\sqrt{2 \pi \mathrm{e}}}\right]\left(1-\frac{x^{2}}{t}\right) \frac{t-t_{0}}{t}+\mathcal{O}\left(\frac{t-t_{0}}{t}\right)^{2}$. 
(We used a series in $s$ of the term in brackets of $u^{(12)}$, and performed the Gaussian integrals.) In fact, these terms in $u^{(12)}$ are irrelevant in the asymptotic limit of large times, and are negligible with respect to $u^{(11)}$ near $t_{0}$, thus we will neglect them in the following. In summary, collecting the contributions to $u$ up to first order we arrive at,

$$
u\left(x, t ; t_{0}\right) \approx Q\left(t_{0}\right) \frac{\mathrm{e}^{-x^{2} / 2 t}}{\sqrt{2 \pi t}}\left[1-\frac{\epsilon}{\sqrt{2 \pi \mathrm{e}}} \ln \frac{t}{t_{0}}\right] .
$$

This is the result of the perturbation expansion in powers of $\epsilon$, and is valid only in a neighborhood of $t \sim t_{0}$, as a consequence of the secular terms. In spite of these limitations, this perturbation expansion is all what we need to apply the renormalization group, using the free parameter $t_{0}$. Therefore, we compute using formula (40) the envelope of the family of trajectories given by Eq. (48), in order to find the form of the global approximation $u(x, t) \approx u\left(x, t ; t_{0}=t_{0}(t)\right):$

$$
0=\left.\frac{d u}{d t_{0}}\right|_{t_{0}=t}=\frac{\partial u}{\partial t_{0}}+\left.\frac{\partial u}{\partial Q} \frac{d Q}{d t_{0}}\right|_{t_{0}=t}
$$

Hence, we deduce the flow equation for the initial conditions,

$$
\dot{Q}+\left.\frac{\alpha}{t_{0}}\right|_{t_{0}=t}=0, \alpha=\frac{\epsilon}{\sqrt{2 \pi \mathrm{e}}} \Rightarrow Q(t)=M / t^{\alpha},
$$

where $M$ is the integration constant. Using this result in the envelope $u\left(x, t ; t_{0}(t)\right)$, we finally obtain the result:

$$
u(x, t) \approx u\left(x, t ; t_{0}(t)\right)=\frac{M}{t^{1 / 2+\alpha(\epsilon)}} \frac{\mathrm{e}^{-X^{2} / 2}}{\sqrt{2 \pi}}, X=\frac{x}{\sqrt{t}},
$$

in accordance with the second kind similarity analysis of Eq. (43), and where the anomalous exponent $\alpha=\epsilon /(2 \pi \mathrm{e})^{1 / 2}$ was computed to the first order in $\epsilon$.

Equation (50) is a kind of amplitude equation, giving a recipe to "optimally" (at order $\epsilon$ ) define the better amplitude at each time, which depends on the scaling factor, such that $u_{\epsilon}$ approaches $u$. The optimal choice corresponds to replacing the unknown exact solution, by the envelope of a one-parameter family of curves, at the given time. The final result is perturbative in the sense that $\left|u-u_{\epsilon}\right| \sim \mathcal{O}\left(\epsilon^{2}\right)$, and simultaneously asymptotic, in the sense that it is valid only for times large enough to "forget" the specific form of the initial state (this is consistent with the neglect of higher order secular terms). 


\section{Shocks, Burgers equation and finite time singularities}

Physical systems develops sometimes singularities, such as shocks and discontinuity surfaces, transitions between states of different topologies, like in a variety of interface phenomena, or in vortex dynamics ${ }^{43}$. Sometimes singularities appear during the evolution of a regular initial state, in a finite time: a physical quantity like acceleration, curvature, pressure, blows up to infinity, signaling perhaps the transition to a new state or the emergence of new characteristic scales, not directly related to external, initial or boundary conditions ${ }^{24}$. Singularities appear in a wealth of physical phenomena as a relevant processes, around which the whole system is organized. In the same way as in the geometrical description of mechanics, using the properties of the phase space trajectories, the global properties of the system can be inferred from the nature of singular points, the evolution of systems undergoing a singular behavior, is determined be the nature of the singularity. We exclude to our analysis "trivial" singularities, arising because of a lack of accuracy in the physical description, and not directly related to the structure of the system. Relevant physical singularities are robust with respect to the addition of regularization processes ${ }^{13}$. In the simple case of a shock wave, a discontinuity in pressure, velocity and density, the propagation properties do not depend on the small scale structure of the shock, determined by dissipation processes (heat, viscosity) ${ }^{29}$. Analogously, when a thread of water breaks into a series of drops, the drop sizes are not directly related to the microscopic effects (van der Waals forces, for example) playing a role during the break up, whereas the topology change (from a connected surface to a multiple connected one) is related to a finite time singularity ${ }^{12}$. In recent years the investigation of singularities in physical systems received a great deal of interest, as for instance in vortex dynamics ${ }^{34},{ }^{30},{ }^{7}$, or in Hele-Shaw cell flows ${ }^{6},{ }^{3}$, or in fluid interfaces ${ }^{12},{ }^{28},{ }^{40},{ }^{41}$.

We start our study of singularities by a simple example taken from the physics of shock waves in compressible flows. Consider the one-dimensional continuity and motion equations of a gas,

$$
\left\{\begin{array}{l}
\rho_{t}+(\rho u)_{x}=0 \\
u_{t}+u u_{x}=-\frac{p_{x}}{\rho}+\nu u_{x x}
\end{array}\right.
$$

where, as usual, $\rho=\rho(x, t), p=p(x, t)$, and $u=u(x, t)$ are the density, pressure and velocity of the fluid; we included the effect of the kinematic viscosity $\nu$, but we assume in the following the limit $\nu \rightarrow 0$. The simplest situation produces for isobaric flows $p_{x}=0$; the velocity equation reduces 
to Burgers' equation ${ }^{44}$ :

$$
u_{t}+u u_{x}=\nu u_{x x}
$$

which in the inviscid limit writes,

$$
u_{t}+u u_{x}=0 .
$$

Equation (54) describes the propagation of simple waves; it is straightforward to verify that the general solution is of the form,

$$
u(x, t)=U(x-u(x, t) t),
$$

by substitution into (54), where $U$ is an arbitrary function of its argument $X=x-u(x, t) t$, that can be associated with the initial condition $u(x, 0)=$ $U(x)$. The nonlinearity of the simple wave equation manifests in the implicit character of (55), indeed, the argument of $U$ contains the unknown $u$, and to obtain an explicit solution one needs to invert $U$. However, inversion is not always possible, in particular, it becomes conceivable that even for a smooth function $U$ the inversion fails at some finite time $t_{*}$, revealing the appearance of some infinite derivative of $u$. Let us compute the spatial derivative of $u$,

$$
u_{x}=\frac{U_{X}}{1+U_{X} t}
$$

which allows us to identify the singularity time $t_{*}$ as the first time for which $U_{X}=-1 / t$, or,

$$
1 / t_{*}=\max _{x}\left\{-U_{X}>0\right\} .
$$

At $t=t_{*}$ the velocity gradient diverges, for $t>t_{*}$ the velocity becomes a multivalued function of $x$, showing that the description based on the simple wave equation ceases to be valid. Using these results for the velocity, we can also compute the density,

$$
\rho=\frac{G(X)}{1+U_{X} t},
$$

where $\rho(x, 0)=\left.G(x-u(x, t) t)\right|_{t=0}$ gives the initial density distribution. We note that at the singularity time $t_{*}$, simultaneously to the divergence in the velocity gradient, the density tends to infinity (provided that $G$ is positive for $t=t_{*}$ ). These are rather esoteric properties for a normal gas, ensuing from the hypothesis of isobaric flow, however they may be relevant for pressureless gases like galaxy systems used in cosmology ${ }^{16}$, or even in some regimes of modified hydrodynamic models undergoing finite time singularities 27 . 
From a mathematical point of view, Eq. (54) pertains to the category of quasilinear partial differential equations, and can be also solved using the method of characteristics. The characteristics of the simple wave equation are $d t / d s=1, d x / d s=u$, and $d u / d s=0$, where $s$ is the parameter of the characteristic line; hence $u(s)=u(x(s), t(s))=u(0)=$ const., on the straight lines $x(s)=x(0)+u(0) s$, and $t=s$ (we put $t=0$, for $s=0$ ). Taking $u(0)=u(x(0), t(0))=U(x(0))=U(x(s)-u(0) s)=U(x(s)-$ $u(s) s)=u(s)$ we arrive at $u=U(x-u t)$. Sometimes it is easier to give the family of characteristic lines, instead of the explicit solution of the quasilinear equation. In the simple wave case, the characteristics are a family of straight lines whose slopes are the initial values of the function $u(x, 0)=U(x)$. It is obvious that if the slope decreases in some $x$ interval for $s=0$, the emerging lines will intersect at some finite $s$, the singularity time, above which the $u$ function is no more uniquely valued.

Let us take the simple profile,

$$
u(x, 0)=\frac{A x}{x^{2}+a^{2}}, \Rightarrow u(x, t)=A \frac{x-u(x, t) t}{(x-u(x, t) t)^{2}+a^{2}},
$$

(with $[A]=\mathrm{L}^{2} \mathrm{~T}^{-1}$ and $[a]=\mathrm{L}$ ), applying Eq. (56) one finds $t_{*}=8 a^{2} / A$. We can see in figure 7 a series of velocity profiles for different times, up to the singularity time, and the corresponding family of characteristic lines (figure 8 ). We note that the $x=0$ point, where the velocity is zero, remains fixed; this happens because $u=0$ is an exact solution of the simple wave equation: if the function $u$ vanishes in some interval it will remain zero at later times on this interval. Moreover, the "interior" region (the region between the origin and the velocity maximum) evolves towards an almost straight line. This remark suggest us that a given initial form, say a bump, will evolve at large times, into a straight line, at least over a finite range, irrespectively of what happens near the breaking front of the wave.

In summary, we obtained an exact solution of the simple wave equation valid up to the singularity time. After this time the nature of the system's evolution remains unknown, but we suspect that the shape of the solution approaches a straight line, and presume that at the breaking point a shock is formed, whose structure should depends on dissipation processes. Therefore, we try to solve the simple wave equation using the method of similarity, in order to investigate a class of asymptotic solutions, valid for times larger than the singularity time $t_{*}$, in the form of localized waves, vanishing outside some interval. We assume that in addition to the differential 


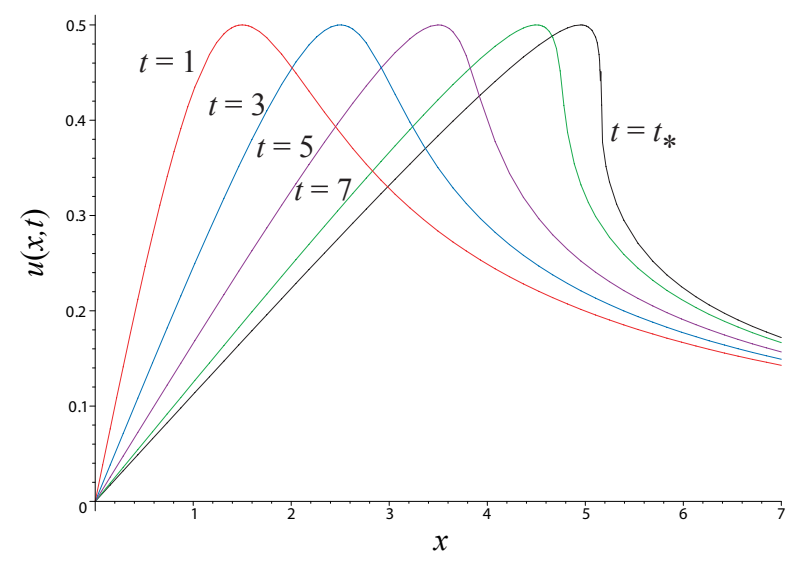

Figure 7. Profiles of the velocity for different times: at $t=t_{*}$ the velocity gradient diverges and the wave breaks. (Only the $x>0$ region is shown, since $u$ is an odd function of $x$; time is measured in units of $a^{2} / A$.)

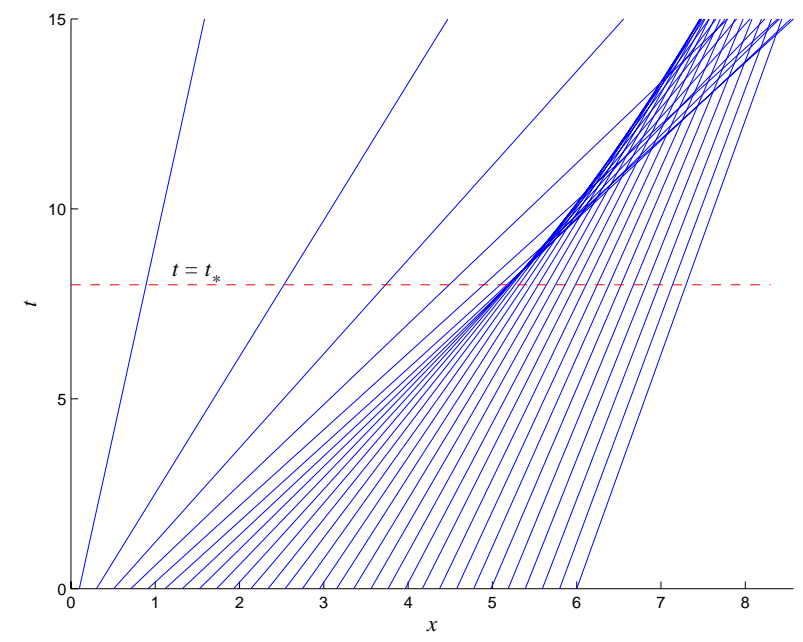

Figure 8. Family of characteristics of the simple wave equation for the initial lorentzian velocity (58). The singularity time occurs at the first intersection of lines at $t=8$ (in units of $a^{2} / A$ ); after this time the envelope of the family forms a caustic line.

equation (54) we have the constraint,

$$
Q=\int_{0}^{R(t)} d x u(x, t)=\text { const. }
$$


related to the conservation law $(d / d t) \int d x u=0$, where $R(t)$ is the shock position, satisfying $u(R(t), t)=0$. The only dimensional parameter of the problem is $[Q]=\mathrm{L}^{2} \mathrm{~T}^{-1}$, hence we seek for a self-similar solution of the form $u=\sqrt{Q / t} U(X)$, with $X=x / \sqrt{Q t}$. This gives the simple solution:

$$
u(x, t)=\left\{\begin{array}{ll}
\frac{x}{t}, & \text { for } 0<x<R(t)=\sqrt{2 Q t} \\
0, & \text { outside }
\end{array},\right.
$$

having the form of a ramp, whose area is constant, and extending in space following the law $R \sim t^{1 / 2}$. The shock velocity is thus $\dot{R}=\sqrt{Q / 2 t}$. This solution, although discontinuous, is physically more satisfactory than the "exact" solution which develops a singularity in finite time, for times much larger that the singularity time.

In order to resolve the structure of the shock, as well as the dynamics of the wave, we return to Burgers equation (53). It is well known that it can be solved using a simple nonlinear transformation which lead to the heat equation ${ }^{44}$. However, it could be more illuminating, and easier, to study a special family of solutions related to the so called "pole decomposition" ${ }^{42}$. One remarkable property of Burgers equation is that it admits the following decomposition,

$$
u(x, t)=-2 \nu \sum_{n=1}^{2 N} \frac{1}{x-z_{n}(t)},
$$

where the pole positions are the complex $z_{n}$ coming by conjugate pairs $(u$ is of course real). Once (60) is inserted into (53), one finds that the poles must move in accordance with the equations,

$$
\dot{z}_{n}=-2 \nu \sum_{k \neq n} \frac{1}{z_{n}-z_{k}}
$$

in order to satisfy the Burgers equation. This condition follows from the identity,

$$
\sum_{n} \sum_{k \neq n} \frac{1}{x-z_{n}} \frac{1}{x-z_{k}}=2 \sum_{n} \sum_{k \neq n} \frac{1}{z_{n}-z_{k}} \frac{1}{x-z_{n}},
$$

as can be demonstrated using an expansion in simple fractions. The simplest solution of the Burgers equation is given by the $N=1$ pair of poles $z(t)=a(t)+\mathrm{i} b(t)$. In this case Eqs. (61) reduce to the real differential equations,

$$
\dot{a}=0, \dot{b}=\frac{\nu}{b}, \Rightarrow a=a_{0}, b=\sqrt{b_{0}^{2}+2 \nu t},
$$


from which one finds,

$$
u(x, t)=-4 \nu \frac{x-a_{0}}{\left(x-a_{0}\right)^{2}+b_{0}^{2}+2 \nu t} .
$$

In the inviscid limit $\nu \rightarrow 0$, this solution describes the formation of a shock around the central position $x=a_{0}$ (the function (62) vanishes for $\nu=0$, except when $\left.x=a_{0}\right)$. Computing the gradient we notice that the maxima locate at $\left|x-a_{0}\right|=\sqrt{2 \nu t}$, from which we deduce that the shock width $\delta$, is of the order of $\delta=\sqrt{2 \nu t}$. This is the usual diffusive behavior related to the term $\nu u_{x x}$ in Burgers equation. Outside the shock region, $\left|x-a_{0}\right| \gg \delta$ the function slowly decays towards zero.

A slightly more rich case is for $N=2$, the superposition of two pairs of symmetric poles with $z_{1}=a+\mathrm{i} b$, and $z_{3}=-a+\mathrm{i} b$. The pole motion equations are:

$$
\dot{a}=\frac{\nu}{a}+\frac{2 \nu a}{a^{2}+b^{2}}, \dot{b}=\frac{\nu}{b}+\frac{2 \nu b}{a^{2}+b^{2}}, \Rightarrow a^{2}+b^{2}=\text { const. }+8 \nu t,
$$

the integration constant is chosen so that $a(0)=a_{0}$ and $b(0)=0$ (this allows us, to avoid dimensional constants, to define $a_{0}$ as the unit of length and $a^{2} / \nu$ as the unit of time). In dimensionless form the solution is then,

$$
a(t)=\sqrt{1+8 t-b(t)^{2}}, b(t)^{2}=\frac{1}{3}\left(1+8 t-(1+8 t)^{1 / 4}\right),
$$

which finally leads to the velocity,

$$
u(x, t)=-4 \frac{x-a(t)}{(x-a(t))^{2}+b(t)^{2}}-4 \frac{x+a(t)}{(x+a(t))^{2}+b(t)^{2}} .
$$

The shape of the velocity (63) is depicted in figure 9. We observe that this solution describes the propagation of two opposite shocks. The shock structure is the same as in the single pair of poles solution. In addition, there is now a central region, far from the shock region, which evolves almost independently of the dissipation mechanism. Indeed, the central region has the asymptotic form $u \approx x / t$, independent of the dissipation constant $\nu$, for $t \gg 1$ and $x \ll \sqrt{t}$. This is precisely the behavior found using the similarity solution.

In conclusion, one may think that a "typical" solution of the simple wave equation, regularized with a small viscosity term, will consists in a series of ramps separated by sharp shocks, where matter concentrates (remember the behavior of the density). This qualitative description coincides with the filamentary distribution of large structures of the universe ${ }^{16}$. It is also worth noticing the independence of the system global behavior, to 
the microscopic scales associated to shock structures: the singularities are genuine in the sense that they organize the large scale shape of the system, and persists in the limit of vanishing viscosity.

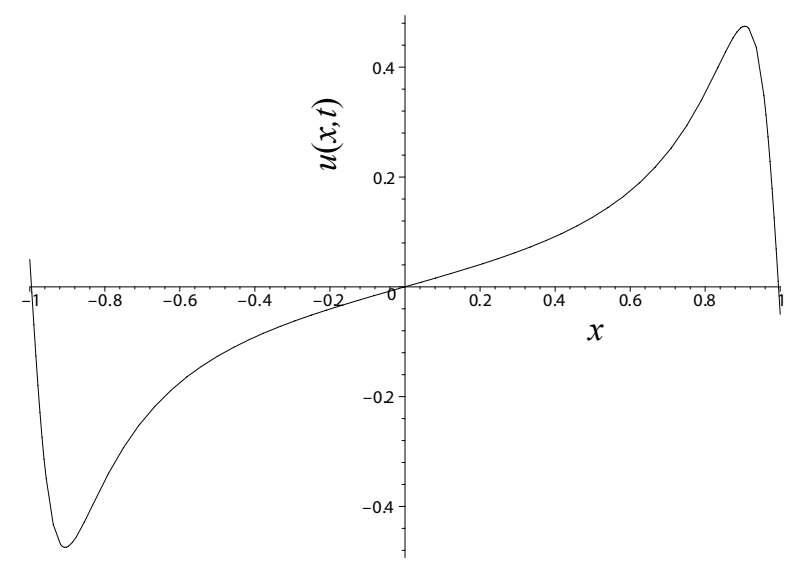

Figure 9. The four poles solution of the Burgers equation: two shocks around $x= \pm 1$ propagates away, leaving a central straight region.

\section{Acknowledgments}

I gratefully acknowledge the organizers of the Peyresq meeting, Jean-Luc Beaumont and Jacques-Alexandre Sepulchre who invited me to give a series of lectures. I warmly thanks the numerous participants who contributed to create a friendly and creative atmosphere. I benefited in particular from discussions with Thomas Frisch, who helped me in preparing this material, and Yves Pomeau who introduced me to this subject.

\section{References}

1. W. F. Ames. Nonlinear Partial Differential Equations in Engineering. Academic Press, New York, 1965.

2. G. I. Barenblatt. Scaling, self-similarity, and intermediate asymptotics. Cambridge University Press, Cambridge, United Kingdom, 1996.

3. A. L. Bertozzi, M. P. Brenner, T. F. Dupont, and L. P. Kadanoff. Singularities and similarities in interface flows. In Lawrence Sirovich, editor, Trends and Perspectives in Applied Mathematics, volume 100 of Applied Mathematical Sciences, pages 155-208. Springer-Verlag, New York, 1994.

4. L.-Y. Chen, N. Goldenfeld, and Y. Oono. Renormalization group theory for global asymptotic analysis. Phys. Rev. Letters, 73(10):1311-1315, 1994. 
5. L.-Y. Chen, N. Goldenfeld, and Y. Oono. Renormalization group and singular perturbations: Multiple scales, boundary layers, and reductive perturbation theory. Phys. Rev. E, 54(1):376-394, 1996.

6. P. Constantin, T. F. Dupont, R. E. Goldstein, L. P. Kadanoff, M. J. Shelley, and S.-M. Zhou. Droplet breakup in a model of the Hele-Shaw cell. Phys. Rev. E, 47(6):4169-4181, 1993.

7. S. J. Cowley, G. R. Baker, and S. Tanveer. On the formation of Moore curvature singularities in vortex sheets. J. Fluid Mech., 378:233-267, 1999.

8. S. H. Davis. Interfacial fluid dynamics. In M. G. Worster G. K. Batchelor, H. K. Moffatt, editor, Perspectives in Fluid Dynamics, pages 1-51, Cambridge, United Kingdom, 2000. Cambridge University Press.

9. P.-G. de Gennes. Wetting: statics and dynamics. Rev. Mod. Phys., 57:827863, 1985.

10. J. A. Diez, R. Gratton, and J. Gratton. Self-similar solution of the second kind for a convergent viscous gravity current. Phys. Fluids, A4(6):1148-1155, 1992.

11. E. B. Dussan and S. H. Davis. On the motion of a fluid-fluid interface along a solid surface. J. Fluid Mech., 65:71-95, 1974.

12. J. Eggers. Nonlinear dynamics and breakup of free-surface flows. Rev. Mod. Phys., 69(3):865-929, 1997.

13. J. Eggers. Hydrodynamic singularities. In J. Parisi, S. C. Mueller, and W. Zimmermann, editors, A Perspective Look at Nonlinear Media. From Physics to Biology and Social Sciences, page 1, Berlin, 1998. Springer. http://arXiv.org/abs/physics/0110087.

14. J. Eggers and H. A. Stone. Characteristic lenghts at moving contact lines for a perfectly wetting fluid: the influence of speed on the dynamic contact angle. arXiv: physics/0210101, 2002.

15. S.-I. Ei, K. Fujii, and T. Kunihiro. Renormalization-group method for reduction of evolution equations; invariant manifolds and envelopes. Annals Phys., 280:236-298, 2000.

16. U. Frisch and J. Bec. "Burgulence". arXiv: nlin.CD/0012033, 2001.

17. L. Giacomelli and F. Otto. Droplet spreading: intermediate scaling law by PDE methods. Comm. Pure Appl. Math., 55:217-254, 2002.

18. N. Goldenfeld. Lectures on Phase Transitions and the Renormalization Group. Addison-Wesley, Reading, Massachusetts, 1992.

19. N. Goldenfeld, O. Martin, Y. Oono, and F. Liu. Anomalous dimensions and the renormalization group in a nonlinear diffusion process. Phys. Rev. Letters, 64(12):1361-1364, 1990.

20. J. Gratton. Similarity and self similarity in fluid dynamics. Fund. Cosmic Phys., 15:1-106, 1991.

21. J. Gratton and F. Minotti. Self-similar viscous gravity currents: Phase-plane formalism. J. Fluid Mech., 210:155-182, 1990.

22. J. Gratton and C. Vigo. Self-similar gravity currents with variable inflow revisited: Plane currents. J. Fluid Mech., 258:77-104, 1994.

23. C. Huh and L. E. Scriven. Hydrodynamic model of steady movement of a solid/liquid/fluid contact line. J. Colloid Interface Sci., 35:85-101, 1971. 
24. L. Kadanoff. Singularities and blowups. Phys. Today, September:11-13, 1997.

25. T. Kunihiro. A geometrical formulation of the renormalization group method for global analysis II: Partial differential equations. Japan J. Ind. Appl. Math., 14:51, 1997.

26. Teiji Kunihiro. A geometrical formulation of the renormalization group method for global analysis. Prog. Theor. Phys., 94:503-514, 1995. Erratum: Prog. Theor. Phys. 95, 835 (1996).

27. E. A. Kuznetsov and V. P. Ruban. Hamiltonian dynamics of vortex and magnetic lines in hydrodynamic type systems. Phys. Rev. E, 61:831-841, 2000.

28. E. A. Kuznetsov, M. D. Spector, and V. E. Zakharov. Formation of singularities on the free surface of an ideal fluid. Phys. Rev. E, 49(2):1283-1290, 1994.

29. L. Landau and E. Lifchitz. Mécanique des Fluides. Mir, Moscou, 1989.

30. A. J. Majda and A. L. Bertozzi. Vorticity and incompressible flow. Cambridge University Press, New York, 2001.

31. P. O'Grady. Thales of Miletus (62?-546 bce). In J. Fieser and B. Dowden, editors, The internet encyclopedia of philosophy, page 1, University of Tennessee, 2001. http://www.utm.edu/research/iep/t/thales.htm.

32. A. Oron, S. H. Davis, and S. G. Bankoff. Long-scale evolution of thin films. Rev. Mod. Phys., 69(3):931-980, 1997.

33. Y. Pomeau. Recent progress in the moving contact line problem: a review. C. R. Acad. Sc., Mécanique, 330:207-222, 2002.

34. P. G. Saffman. Vortex Dynamics. Cambridge University Press, London, 1992.

35. L. Sedov. Similitude et Dimensions en Mécanique. Mir, Moscou, 1972.

36. M. Serres. Éléments d'Histoire des Sciences. Bordas, Paris, 1989.

37. J. R. Spitzer. Physics of Fully Ionized Gases. Wiley, Interscience, New York, 1956.

38. L. H. Tanner. The spreading of silicone oil dorps on horizontal surfaces. J. Phys. D: Appl. Phys., 12:1473-1484, 1979.

39. R. Taton. La science antique et médivale. Presses Universitaires de France, Quadrige, Paris, 1994.

40. A. Thess, D. Spirn, and B. Jüttner. Viscous flow at infinity Marangoni number. Phys. Rev. Letters, 75(25):4614-4617, 1995.

41. A. Thess, D. Spirn, and B. Jüttner. A two-dimensional model for slow convection at infinite Marangoni number. J. Fluid Mech., 331:283-312, 1997.

42. O. Thual, U. Frisch, and M. Hénon. Application of pole decomposition to an equation governing the dynamics of wrinkled flame fronts. J. Physique, 46:1485-1494, 1985.

43. A. Verga. Singularity formation in vortex sheets and interfaces. In E. Tirapegui and S. Martínez, editors, Nonlinear Phenomena and Complex Systems, pages 1-18. Kluwer, Amsterdam, 2003, to appear. http://www.irphe.univ-mrs.fr/ verga/Archive/proc.pdf.

44. G. B. Whitham. Linear and Nonlinear Waves. Wiley, New York, 1974.

45. J. Zinn-Justin. Quantum Field Theory and Critical Phenomena. Clarendon Press, Oxford, 2002. 